\title{
Approches de l'influence des parents sur le développement vocationnel des adolescents
}

Parental influence on career development in adolescents

Helena Rebelo Pinto et Maria da Conceição Soares

\section{OpenEdition}

1 Journals

Édition électronique

URL : http://journals.openedition.org/osp/2272

DOI : 10.4000/osp.2272

ISSN : 2104-3795

Éditeur

Institut national d'étude du travail et d'orientation professionnelle (INETOP)

Édition imprimée

Date de publication : 15 mars 2004

Pagination : 7-24

ISSN : 0249-6739

Référence électronique

Helena Rebelo Pinto et Maria da Conceição Soares, « Approches de l'influence des parents sur le développement vocationnel des adolescents », L'orientation scolaire et professionnelle [En ligne], 33/1 | 2004, mis en ligne le 22 octobre 2009, consulté le 19 avril 2019. URL : http:// journals.openedition.org/osp/2272; DOI : 10.4000/osp.2272

Ce document a été généré automatiquement le 19 avril 2019

(c) Tous droits réservés 


\title{
Approches de l'influence des parents sur le développement vocationnel des adolescents
}

Parental influence on career development in adolescents

\author{
Helena Rebelo Pinto et Maria da Conceição Soares
}

1 L'influence des parents sur le développement vocationnel de leurs enfants est reconnue par différents modèles théoriques et dans les pratiques d'aide à l'orientation (Pinto \& Soares, 2001, 2002b). Elle a donné lieu à un plan de recherche au Portugal, conduit par les auteurs, dans le cadre de la Faculté de Psychologie et d'Éducation et de l'Institut d'Orientation Professionnelle de l'Université de Lisbonne.

2 Le plan comportait trois étapes. La première, qui suivait une approche théorique développementale fondée sur le modèle de la maturité vocationnelle de Donald Super (Super \& Thompson, 1979), a conduit à la construction d'un questionnaire à réponses fermées relatif à la connaissance des besoins de formation des parents (Soares \& Pinto, 1994) et a permis une analyse quantitative des résultats obtenus (Soares, 1998; Soares \& Pinto, 1997). Tenant compte des problèmes psychométriques que soulevait l'instrument et des limitations induites par le modèle retenu pour étudier ce phénomène, une deuxième étape a tenté une approche fondée sur une méthodologique qualitative : des entretiens de recherche avec quelques parents d'élèves ont permis de construire un plan d'entretien semi-directif, afin de permettre une analyse élargie et approfondie des perspectives parentales relatives au développement vocationnel de leurs enfants (Pinto \& Soares, 2000). La troisième étape a été conduite en fonction des résultats de l'analyse des données d'entretien ainsi obtenues, en suivant une approche plutôt inductive, interprétées selon les perspectives de la théorie de l'action proposées par Young et collaborateurs (Young \& Friesen, 1990 ; Young, Valach \& Collin, 1996, 2002).

3 L'article présente d'abord quelques références relatives, soit aux perspectives théoriques sur l'influence parentale, soit aux principales voies de recherche empiriques suivies dans 
différents pays, soit encore aux pratiques adoptées pour la formation des parents dans ce domaine. Il décrit ensuite plus en détail les trois étapes des recherches portugaises, en essayant de mettre en relief les procédures suivies, ainsi que les principaux résultats qui ont contribué, au point de vue conceptuel, au progrès de la connaissance du phénomène et, au point de vue des pratiques d'aide à l'orientation, à la mise au point d'une méthodologie de conseil et de formation de parents.

\section{Influence parentale : théorie, recherche et pratique}

4 L'influence de la famille, en particulier l'influence des parents dans le déroulement de la carrière de leurs enfants, est reconnue, de façon plus ou moins affirmée, par les différents modèles théoriques de la psychologie de l'orientation (Bordin, 1990; Gottfredson, 1981, 1996 ; Holland, 1959,1973 ; Krumboltz, 1996； Lent, Brown \& Hackett, 1996 ; Lofquist \& Dawis, 1969; Roe, 1957; Roe \& Lunneborg, 1990 ; Super, 1957, 1980,1990; Vondracek, 1990; Vondracek \& Fouad, 1994). Au cours des années cinquante, Super et Holland relevaient ce phénomène et Anne Roe le plaçait au centre de son modèle. Par la suite, et dans le cadre de différentes inspirations théoriques, structurales, dynamiques, développementales, cognitives ou sociales, les variables familiales ont été considérées par différents auteurs. Plus récemment, on trouve une approche théorique plus spécifique du phénomène, soit dans le cadre des perspectives systémiques (Bratcher, 1982 ; Blustein, Devenis \& Kidney, 1989 ; Lopez \& Andrews, 1987), soit dans le cadre contextualiste (Palmer \& Cochran, 1988; Vondracek, 1990 ; Vondracek \& Fouad, 1994), ou dans la théorie de l'action contextuelle (Young \& Friesen, 1990 ; Young, Valach \& Collin, 1996, 2002).

Malgré l'importance des variables familiales dans le développement vocationnel, reconnue aussi bien par les jeunes eux-mêmes que par les conseillers d'orientation dans leurs pratiques, il a fallu attendre les années quatre-vingts pour trouver dans la littérature scientifique de l'orientation des études spécifiques sur le sujet, menées généralement dans une perspective strictement empirique (Fouad, 1994). Plusieurs recherches ont ainsi été conduites. Elles mettent en relief les variables familiales les plus directement liées au développement vocationnel des enfants et des adolescents et, tout particulièrement, le niveau socio-économique et culturel (Schulenberg, Vondracek \& Crouter, 1984), la composition de la famille (Blake, 1985), l'origine ethnique (Frost \& Diamond, 1979), les pratiques éducatives (Young, Friesen \& Pearson, 1988), les valeurs familiales (Vodanovich \& Kramer, 1989), l'encouragement parental (Astone \& Mclanahan, 1991), l'interaction parents/enfants (Penick \& Jepsen, 1992), l'action intentionnelle (Young, Friesen \& Dillabough, 1991; Young \& Friesen, 1992; Young, Friesen \& Borycki, 1994). Des études plus récentes considèrent comme des dimensions de l'influence parentale, soit les activités des parents relatives au développement vocationnel de leurs enfants, soit le rôle joué par la dynamique familiale dans ce développement (Young et al., 2001).

La littérature sur l'influence parentale témoigne aussi de l'importance attribuée à la formation des parents dans ce domaine. Différentes études présentent plusieurs programmes et divers moyens d'intervention qui se centrent soit sur des activités d'information sur les opportunités scolaires et professionnelles (Amatea \& Cross, 1980), soit sur des formations visant à donner un rôle plus actif aux parents dans le développement vocationnel (Stevens, 1989). Plus récemment, ont été proposées des 
activités inspirées des perspectives d'interaction parents/ enfants (Cochran, 1985a, 1985b, 1985c ; Cochran \& Amundson, 1985 ; Young, 1994). Parmi celles-ci, on peut citer l'approche du développement vocationnel considéré comme un projet familial (Young et al., 2001) et le recours à des techniques d'auto-confrontation utilisant des enregistrements vidéos, dans le but d'aider les participants à mener des actions conjointes et à clarifier leurs rôles dans la construction de projets scolaires et professionnels (Young, Valach, Ball, Turkel \& Wong, 2002).

7 Bref, au cours des années, le thème de l'influence parentale est devenu de plus en plus saillant dans le domaine de la psychologie de l'orientation, à la fois dans les théories, dans les recherches empiriques et dans les pratiques. Osipow remarque à ce propos (1994) que l'influence parentale peut être vue aujourd'hui comme un exemple de convergence théorique des années quatre-vingt-dix, dans la mesure où différents modèles considèrent les variables familiales dans l'étude des projets d'avenir des jeunes. Il semblerait ainsi que son observation initiale (Osipow, 1983), selon laquelle l'importance attribuée à ce phénomène n'aurait pas conduit à des formulations plus spécifiques, ne soit-fort heureusement - pas restée lettre morte...

\section{Études portugaises}

\section{Première étape - Maturité vocationnelle et influence parentale}

$8 \mathrm{Au}$ début du premier temps des études portugaises concernant l'influence parentale (entre 1994 et 1997), on a pris comme point de départ le modèle de la maturité vocationnelle de Donald Super (Super \& Thompson, 1979) avec, notamment, ses dimensions : attitudes de planification et d'exploration, connaissances sur la prise de décision, la carrière et le monde du travail. On a jugé utile d'y introduire une cinquième dimension, nommée connaissance de l'enfant, qui peut être rapprochée de la dimension de Super " orientation vers la réalité ", compte tenu de l'importance du thème à la fois dans la littérature scientifique et dans les pratiques d'aide à l'orientation.

9 Pour chacune de ces dimensions, on a créé un ensemble d'items, sous la forme de propositions qu'on demandait aux parents d'évaluer sur des échelles à quatre degrés, concernant ou bien leur participation effective, ou bien l'importance qu'ils attribuaient à chacun des aspects considérés. À la suite de différentes procédures visant à améliorer cet outil, un questionnaire comportant deux échelles - Importance et Participation - a été construit, chacune d'elle comprenant 15 items (3 pour chaque dimension). Le questionnaire a été rempli par un échantillon de 90 parents d'élèves de la $9^{\mathrm{e}}$ année de scolarité de l'enseignement public, de la région de Lisbonne, au début d'une séance d'information sur le système éducatif. L'âge moyen des participants était de 41,7 ans. Plus de $80 \%$ avaient fréquenté l'école pendant au moins six ans. Tous habitaient avec leur conjoint et leurs enfants (un, deux, ou trois).

10 L'échelle Importance a été abandonnée vu la tendance des sujets à donner des réponses socialement souhaitables. L'analyse factorielle de l'échelle Participation a permis d'identifier quatre facteurs qui expliquent $60,79 \%$ de la variance totale des résultats: deux facteurs cognitifs et deux facteurs d'attitudes (voir tableau 1). Le premier facteur, qui explique $21,80 \%$ de variance, est un facteur d'information, soit sur la carrière et le monde $\mathrm{du}$ travail, soit sur l'enfant lui-même. Le deuxième facteur, qui explique $16,16 \%$ de variance, concerne aussi l'information, mais celle-ci est plus orientée vers l'enfant lui- 
même, dans une perspective de connaissance de soi. Le troisième et le quatrième facteur (expliquant, respectivement, $13,43 \%$ et 9,40\% de variance) se rapportent plutôt au domaine des attitudes, notamment à ce qui concerne l'accessibilité à des expériences et à des sources d'information ainsi que l'autonomie des enfants.

L'engagement des parents dans des activités d'information se révèle plus marqué chez les parents ayant bénéficié d'une scolarité plus longue, surtout lorsqu'ils sont parents de garçons; ces garçons présentant des résultats plus élevés sur les dimensions d'attitudes du C.D.I., Career Development Inventory (Thompson \& Lindeman, 1984) ${ }^{1}$. Les résultats les plus élevés en matière d'autonomie correspondent aux parents les plus jeunes et ayant plus d'un enfant (Soares, 1998).

\section{Tableau 1/Table 1}

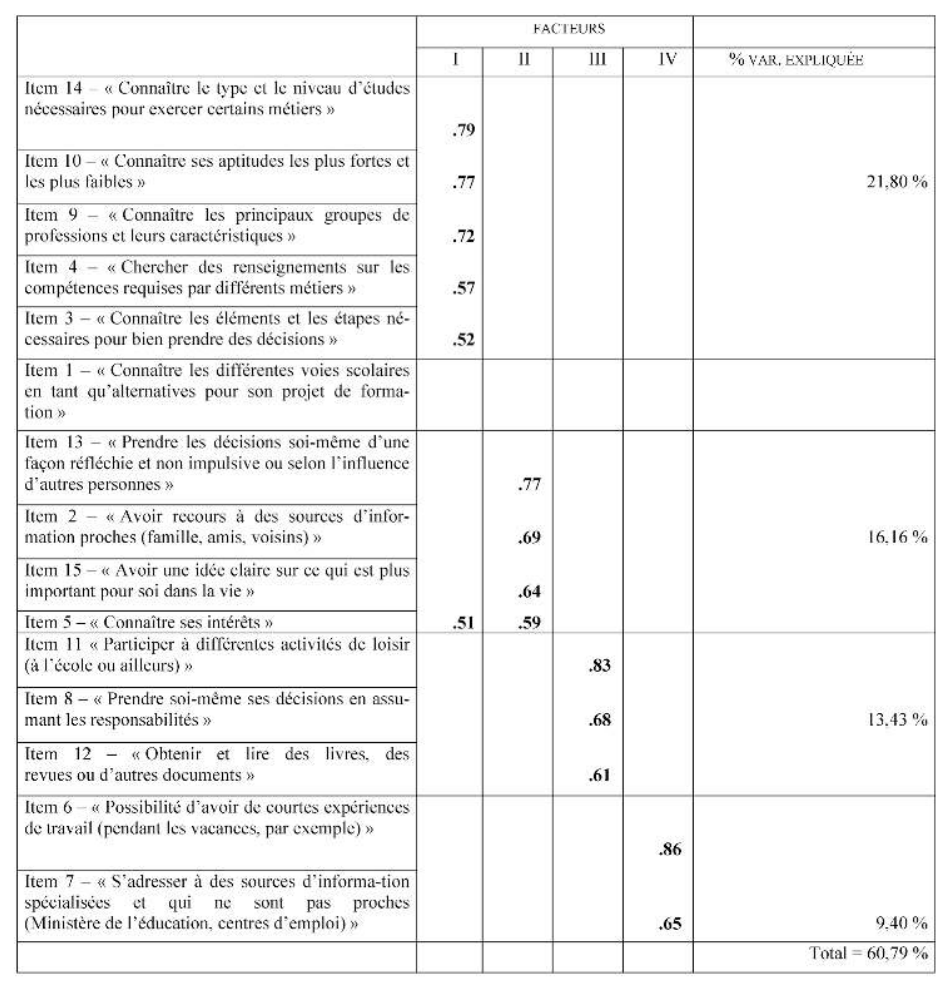

Analyse factorielle des items de l'échelle Participation ( $N=90)$

Factorial analysis of participation scale items

En résumé, ces résultats suggèrent que la participation desparents dans le développement vocationnel de leurs enfants, telle qu'ils l'estiment, s'organise selon deux axes principaux: d'une part, l'information sur les opportunités scolaires et professionnelles et la connaissance de l'enfant et, d'autre part, certaines attitudes concernant l'autonomie de l'enfant et l'accès à des expériences utiles à son développement vocationnel. L'interprétation psychologique de ces résultats, bien que confirmant la coexistence de dimensions cognitives et affectives, signalées dans le modèle de la maturité vocationnelle de Donald Super, révèle certains points de dissonance avec celui-ci qui soulèvent la question de son applicabilité à l'étude du phénomène de l'influence parentale.

13 Ainsi, une analyse qualitative exploratoire du discours de ces mêmes parents au cours d'entretiens de conseil (que l'on avait enregistrés), menée en complément de l'étude 
quantitative, a révélé des éléments clairement reliés au sens psychologique attribué aux facteurs, mais a aussi témoigné de la présence d'autres thèmes dans leurs expériences, leurs préoccupations et leurs pratiques éducatives concernant le développement vocationnel de leurs enfants. Par exemple, les parents s'interrogent sur l'importance de l'information sur les métiers ou sur la mise en valeur de la connaissance de l'enfant, en exprimant des perspectives, souvent contradictoires, à propos de son autonomie, à la fois souhaitée et crainte, surtout quand il s'agit de favoriser l'accès à des expériences nouvelles. Dans leur discours, les parents se reconnaissent un rôle important dans le développement vocationnel des enfants tout en se rendant toutefois compte qu'ils auraient besoin de soutien pour le jouer d'une façon plus efficace. C'est surtout le cas de parents présentant des résultats quantitatifs faibles au questionnaire utilisé. D'autre part, et à un niveau d'analyse plutôt interprétatif, on a souvent repéré dans les discours des parents des éléments concernant leur propre expérience, leurs valeurs, leurs projets ou leurs rêves, qu'ils attribuaient à leurs enfants, ne se rendant même pas compte qu'ils le faisaient (Soares, 1998).

Par la suite, une nouvelle voie de recherche s'est ouverte, à partir d'une méthodologie qualitative plus proche des expériences des parents, de leurs pensées et de leurs sentiments, à propos des projets et des opportunités scolaires et professionnelles de leurs enfants. C'est cette deuxième étape des études portugaises sur l'influence parentale que l'on décrit maintenant.

\section{Deuxième étape - Importance et participation parentale au développement vocationnel}

Dans le but d'élargir et d'approfondir le cadre conceptuel et méthodologique pour l'étude de l'influence parentale, on a réalisé huit entretiens de recherche avec des parents appartenant à l'échantillon de la première étude. On a choisi ces parents en fonction de différentes combinaisons de résultats quantitatifs élevés et faibles dans les deux échelles du questionnaire utilisé dans cette première étude, dans le but d'assurer à l'analyse une plus grande diversité de situations. Les entretiens ont été présentés comme un moyen de clarifier et d'approfondir le sens et les expressions du rôle desparents dans le développement vocationnel de leurs enfants; ils ont été conduits et enregistrés par les auteurs.

La lecture flottante des textes des interviews, dans un premier temps, suivie d'une lecture transversale des mêmes textes, dans le but de trouver des régularités dans les discours, ouvrant une première voie de catégorisation (Poirier, Clapier-Valladon \& Raybaut, 1993), ont permis d'identifier dans le discours des parents à propos de leurs enfants de nombreux éléments se rapprochant des résultats d'autres recherches sur l'influence parentale. On a donc décidé de suivre une approche plutôt déductive dans l'analyse de contenu des textes, en essayant de s'appuyer sur ces éléments pour la formation de catégories.

Les textes de la transcription ont d'abord été soumis à une analyse de contenu selon les deux axes qui avaient inspiré le questionnaire : importance attribuée par les parents aux différents aspects du développement vocationnel; participation effective des parents à des activités favorisant ce développement. Pour la construction des catégories se rapportant à chaque thème, on a pris des éléments mis en évidence par différents experts dans le domaine de l'influence parentale. En ce qui concerne le thème participation, ont 
été considérées deux catégories a priori selon la typologie de Jacobsen (1971) - dialogues et pratiques familiales et développement vocationnel des enfants. En ce qui concerne le thème importance, on a pris des éléments vus par différents auteurs comme ayant une forte influence sur le développement vocationnel, notamment: la connaissance de l'enfant (McDaniels \& Hummel, 1984); le soutien à la vie scolaire (Astone \& Mclanahan, 1991) et à la construction de projets pour l'avenir (Birk \& Blimline, 1984) ; la coopération avec d'autres agents éducatifs (Hoyt, 1984); la socialisation dans différents contextes (Young \& Friesen, 1992) ; l'interaction familiale (Young, 1994). À partir de ces éléments, on a créé sept catégories a priori qu'on a appliquées à l'analyse du contenu des interviews. Ces procédures ont permis d'identifier, pour chaque thème, les catégories qui se présentaient comme noyaux sémantiques des discours des parents et dans lesquelles on repérait plusieurs sous-catégories. On a abouti à une première grille de catégorisation qui a été soumise à l'appréciation de trois juges indépendants, ce qui a conduit à quelques modifications pour arriver à un niveau d'accord acceptable, en ce qui concerne la structure de l'ensemble et les spécifications, permettant un système de codification suffisant et précis.

18 Concernant le thème Participation, deux catégories ont été identifiées. La première catégorie, que l'on a appelée communication, correspond aux conversations des parents avec leurs enfants, à l'initiative des uns ou des autres, intentionnelles ou non intentionnelles, sur des sujets liés à la vie scolaire et à l'avenir professionnel. Les unités qui constituent cette catégorie sont nombreuses et on les trouve dans tous les entretiens, sous formes diverses, selon l'initiative, le contenu ou l'intention des dialogues familiaux. La deuxième catégorie, que l'on a nommée activités, correspond aux initiatives des parents et surtout à leur soutien aux initiatives des enfants, traduites en expériences concrètes, dans le but d'obtenir de bons résultats dans la vie scolaire et de bien préparer l'avenir professionnel. Leur incidence est cependant beaucoup moins forte dans le discours des parents et concerne principalement le soutien de la vie scolaire (aide à l'étude, cours divers...), des activités de loisirs (culture, sport...), ou des expériences concernant des prises de décisions. Ces résultats vont dans le sens des conclusions de la première étape de l'étude, c'est-à-dire que l'on retrouve l'importance de ce sujet, qui se manifeste par les conversations entre parents et enfants; mais on se rend compte que les parents ne s'engagent pas tellement dans des activités concrètes dirigées de façon intentionnelle vers le développement vocationnel.

Pour le thème Importance, l'analyse de contenu s'est montrée beaucoup plus riche. On a accepté comme point de départ pour construire les catégories que le fait de les trouver dans les discours des parents sur leur expérience témoignait de l'importance qui leur était attribuée, soit sous forme de préoccupations, soit sous forme de pratiques éducatives. Sept catégories ont été construites. La première catégorie, que l'on a appelée connaissance de l'enfant, comprend les éléments du discours des parents concernant les caractéristiques personnelles et leur rôle dans le développement vocationnel (aptitudes, intérêts, valeurs, traits de personnalité, santé, etc.). La deuxième catégorie, que l'on a nommée socialisation, a été définie comme préoccupations et pratiques éducatives concernant la dimension sociale du développement (l'intégration de l'enfant dans des groupes qu'on considère utiles à son développement, les relations d'amitié ou de voisinage, l'ouverture de la famille à l'extérieur en même temps que la protection face aux risques de l'autonomie). La troisième catégorie, que l'on a appelée réussite scolaire, a été définie comme préoccupations et pratiques éducatives concernant l'enfant en tant 
qu'élève (notes scolaires, problèmes d'apprentissage, organisation de l'étude, développement de capacités). La quatrième catégorie, que l'on a nommée avenir professionnel, a été définie comme préoccupations et pratiques éducatives concernant les projets professionnels de l'enfant lui-même, les projets des parents pour l'enfant, des évaluations selon des critères de congruence enfant/projet, d'opportunité, de convenance ou de prestige. La cinquième catégorie, que l'on a appelée loisirs, a été définie comme préoccupations ou pratiques éducatives concernant les activités sportives, culturelles, communautaires et religieuses de l'enfant, dans différents cadres institutionnels, envisagées par les parents dans deux perspectives : comme éléments utiles de formation globale de l'enfant, comme opportunités d'exprimer et de développer son identité. La sixième catégorie, que l'on a nommée interaction familiale, a été définie comme préoccupations et pratiques éducatives concernant la situation de l'enfant dans la famille par rapport aux autres membres, l'influence de l'organisation de la vie familiale et du climat affectif, l'importance du dialogue. On a encore identifié une septième catégorie, que l'on a appelée famille/école et qui a été définie comme préoccupations et pratiques concernant le dialogue et la coopération des parents avec les services d'orientation et les professeurs.

20 L'analyse de contenu a encore permis d'identifier deux dimensions transversales aux catégories précédentes. La première, qu'on a appelée expectatives, correspond aux attentes et aux évaluations de probabilités bien présentes dans le discours des parents, concernant soit le présent, soit l'avenir de l'enfant, d'un point de vue plus optimiste ou plus pessimiste. La deuxième dimension concerne le type de discours des parents, tantôt descriptif, tantôt explicatif, voire interprétatif ou intentionnel.

21 En fonction des résultats de cette analyse, on a jugé possible de poursuivre dans cette voie de recherche d'une façon plus structurée et avec des échantillons plus larges. C'est la troisième étape des études portugaises que l'on présente maintenant.

\section{Troisième étape - Action parentale et développement vocationnel}

\section{Instrument}

On a d'abord construit un plan d'interview semi-directif dont l'objectif a été défini en termes de description et d'interprétation de l'action desparents concernant le développement vocationnel des adolescents. Le plan contient trois thèmes, à savoir : communication, activités et expectatives; les deux premiers correspondant aux catégories identifiées dans le cadre de la participation; le troisième découlant de la dimension transversale repérée. Pour chaque thème on a formulé deux questions: la première d'incidence descriptive ; la deuxième, d'incidence explicative. Après quelques reformulations et leur évaluation par quatre experts, les thèmes ont été présentés sous forme de trois questions doubles :

1. «De quoi parlez-vous avec votre enfant concernant ses projets d'avenir et son orientation future? Et pourquoi en parlez-vous?»;

2. «Quelles activités menez-vous dans votre vie familiale pour contribuer à ce développement ? Pourquoi et dans quel but?»;

3. «Quelles sont vos attentes concernant l'avenir scolaire et professionnel de votre enfant? Pourquoi avez-vous ces attentes?». 
Les catégories obtenues dans l'analyse précédente dans le cadre du thème importance, ont été prises comme réponses probables aux questions formulées. Le plan d'interview a été l'objet d'un essai et a été soumis à l'examen de quatre experts en orientation (Pinto \& Soares, 2002a).

\section{Participants}

Le plan a été appliqué à 64 participants, entre 35 et 50 ans, appartenant à différents groupes professionnels $; 27$ parents d'élèves de la $6^{e}$ année de scolarité et 37 parents d'élèves de $9^{e}$; parents de 39 garçons et 25 filles, étudiant dans des écoles publiques de cinq régions du pays. Les participants avaient fréquenté l'école douze ans ou plus.

\section{Procédures}

Les interviews ont été conduites par des psychologues conseillers d'orientation et par des professeurs responsables de classe qui avaient suivi une formation menée par les auteurs sur le thème Influence parentale et développement vocationnel. Le programme de cette formation, d'une durée de 25 heures pour des groupes de 18 participants, était composé de quatre éléments: approches théoriques sur influence parentale et développement vocationnel; analyse d'études empiriques concernant les variables les plus pertinentes dans ce domaine; exemples de programmes de formation de parents; essai et entraînement à l'usage d'une méthodologie de recherche/intervention.

On a conduit l'analyse du contenu de ces entretiens, selon une approche plutôt inductive, dans le but de décrire les expériences des parents à propos, soit de leur influence, soit de leurs perspectives concernant le parcours scolaire et professionnel des enfants. Pour organiser l'information de façon à lui trouver de la cohérence et du sens vis-à-vis de l'objet de la recherche, on a suivi les quatre étapes de la méthodologie d'analyse catégorielle, selon Bardin (1977), à savoir : a) lecture flottante de l'information; b) définition de thèmes et des unités d'information; c) construction d'un système de catégories selon une approche inductive; d) validation de ces catégories.

D'après la lecture globale des 64 entretiens, on a pu identifier un certain nombre de grands thèmes qui ont conduit à une première codification de l'information et à la construction d'une structure capable d'intégrer les données. L'organisation de l'information d'après ces thèmes a permis la division des données en unités d'enregistrement qui, bien que dissociées des groupes auxquels elles appartenaient, gardaient leur sens dans le contexte du corpus. À l'issue de cette organisation logique, les unités d'enregistrement ont été prises en tant qu'unités d'information, toutes codifiées par rapport à leur origine dans les discours des parents. Le regroupement des unités d'information en fonction des grands thèmes qui avaient été identifiés s'est alors déroulé dans un cycle de construction, adaptation et reconstruction - étape fondamentale de systématisation de l'information, permettant d'avancer dans la méthodologie de catégorisation - les catégories étant définies en fonction des groupements logiques des unités de chaque thème. L'application des catégories au traitement de l'information a permis non seulement l'émergence de sous-groupes de plus en plus spécifiques, mais aussi, dans le cadre d'une logique bi-directionnelle, l'émergence de groupements plus larges. On est enfin arrivé à un schéma d'analyse intégrant thèmes, catégories, sous- 
catégories et indicateurs, qui permettent, dans leur ensemble, une approche interprétative des données.

La validation a suivi une méthode d'objectivation des catégories à travers l'intersubjectivité de trois experts participant à l'analyse, suivant des procédures de comparaison et de redéfinition de catégories, dans un premier et un second temps, complétées par l'application des catégories à l'organisation finale des données, dans un troisième temps, par un expert dans le domaine de l'étude. La validation a suivi aussi une méthode de comparaison à des catégories conceptuelles, soit d'origine théorique, soit d'origine empirique (Pinto \& Soares, 2002a).

\section{Résultats}

On a identifié six thèmes dans le discours des participants: trois thèmes découlant directement des réponses aux questions posées aux parents, à savoir :

- communications parents/enfants ;

- interventions familiales ;

- expectatives des parents ;

et trois thèmes obtenus par de nouvelles procédures de catégorisation des réponses obtenues :

- rôles des parents ;

- facteurs d'influence sur le développement vocationnel ;

- perspectives des enfants vues par les parents.

Le tableau 2 présente les catégories et les sous-catégories correspondant à chaque thème, chaque sous-catégorie étant accompagnée d'un exemple de discours.

Le thème communication parents/enfants découle directement des réponses aux questions, et comprend trois catégories : l'initiative (soit des parents eux-mêmes, soit des enfants), le contenu (concernant l'école, des thèmes d'actualité, les métiers, les problèmes d'adolescence), et l'intention (concernant soit la connaissance de l'enfant, soit l'aide à son développement, soit l'orientation éducative ou professionnelle). Le thème interventions familiales concerne les activités conduites par les parents dans le but d'aider et d'influencer leurs enfants, groupées en deux catégories : activités spécifiques (appui au travail scolaire, au projet vocationnel, à l'orientation professionnelle) et structure de situations (concernant le développement personnel, l'organisation de la vie familiale, les opportunités et les ressources). Le thème expectatives des parents concerne les désirs et les aspirations des parents, pour un avenir plus proche ou plus lointain, groupés en trois catégories: l'enfant en tant que personne (identité, valeurs, intégration sociale, réalisation personnelle), l'enfant en tant qu'élève (projets, niveau d'études, réussite scolaire, attitude face à l'école) et l'enfant en tant que professionnel (emploi satisfaisant, réussite professionnelle, groupe professionnel).

Le thème rôle des parents a été construit à partir de l'organisation des unités d'information dans lesquelles les participants parlent de ce que les parents, en général, doivent faire concernant la carrière des enfants. Ce thème intègre trois catégories : conseiller (soit de leur propre initiative, soit en réponse à la demande de l'enfant), appuyer (acceptant les choix de l'enfant, offrant des moyens), motiver (se montrer disponible et intéressé, faire confiance). Le thème facteurs d'influence concerne les éléments envisagés par les parents comme ayant une forte influence sur les projets de 
leurs enfants: facteurs personnels (aptitudes, intérêts, valeurs, expériences scolaires, maturité, style relationnel, attitudes), agents éducatifs (soit les parents eux-mêmes, soit d'autres membres de la famille, des professeurs, des conseillers d'orientation) et conditions de milieu (système éducatif, emploi, situation familiale).

Le dernier thème concernant les perspectives des enfants sur le développement vocationnel, vues par les parents eux-mêmes, a été identifié par inférence et non comme élément du discours. En fait, tout en exprimant dans leurs discours le respect de l'autonomie, les parents essaient souvent de saisir les perspectives des enfants sur l'avenir professionnel pour soutenir leurs propres expectatives. On a organisé ce thème en trois axes : l'enfant lui-même, les parents et la société. Ces catégories indiquent qu'en plus des éléments concernant l'enfant, que les parents semblent reconnaître et accepter, l'interprétation parentale est toujours présente, se rapportant soit à des objectifs personnels, soit à des valeurs sociales (Pinto \& Soares, 2002a).

Tableau 2/Table $2(1 / 3)$

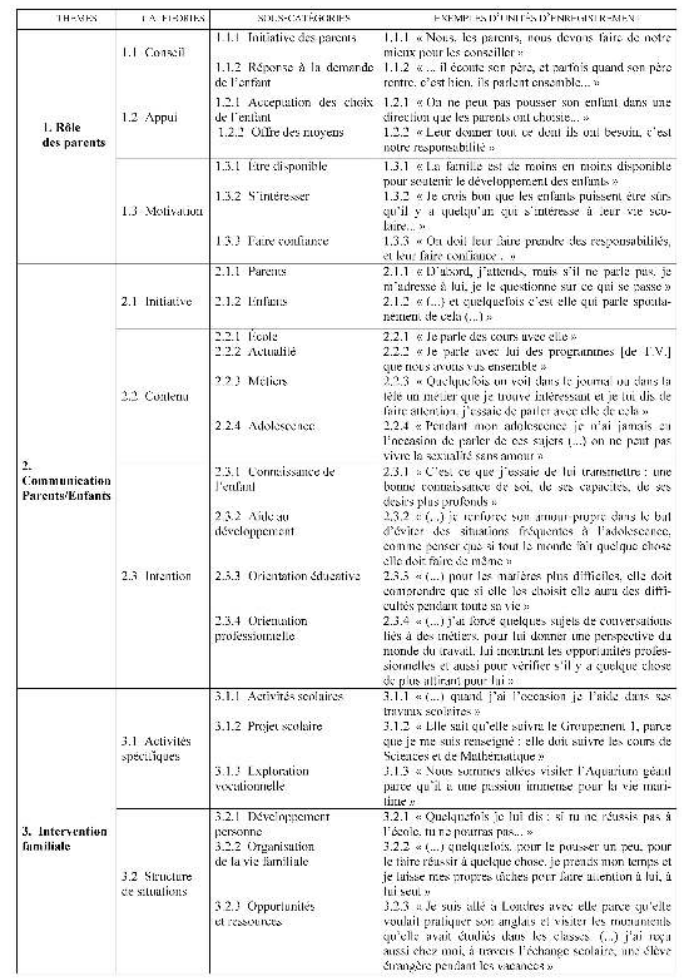

Grille de catégorisation des entretiens avec les parents (1/3) Parent interview categorization 
Tableau 2/Table $2(2 / 3)$

\begin{tabular}{|c|c|c|c|}
\hline \multirow[t]{3}{*}{$\begin{array}{l}\text { 4. Facteurs } \\
\text { d'influence }\end{array}$} & 4,1 Fnlant & $\begin{array}{l}\text { 4.1.4 Hxpériences scolaires } \\
\text { 4.1.5 Maturité } \\
\text { 4.1.6 Style relationnel } \\
\text { 4.1.7 Attitudes }\end{array}$ & 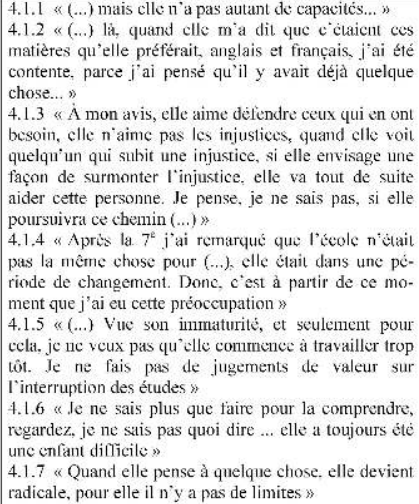 \\
\hline & $\begin{array}{l}\text { 4.2 Agents } \\
\text { éducuatifs }\end{array}$ & $\begin{array}{l}\text { 4.2.1 Parents } \\
\text { 4.2.2 Mcmbres de la fanille } \\
\text { 4.2.3 Professeurs } \\
\text { 4.2.4 Conseillers } \\
\text { d' orientation }\end{array}$ & 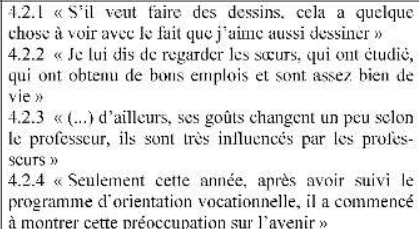 \\
\hline & $\begin{array}{l}4.3 \text { Conditions } \\
\text { du milieu }\end{array}$ & $\begin{array}{l}\text { 4.3.1 Système éducatif } \\
\text { 4.3.2 Emploi } \\
\text { 4.3.3 Situation familiale }\end{array}$ & 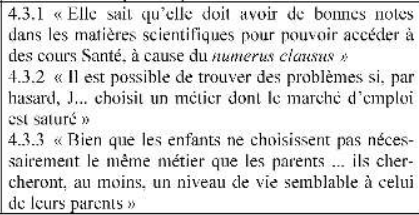 \\
\hline
\end{tabular}

Grille de catégorisation des entretiens avec les parents $(2 / 3)$

Parent interview categorization 


\begin{tabular}{|c|c|c|c|}
\hline \multirow{3}{*}{$\begin{array}{l}\text { 5. Expectatives } \\
\text { des parents }\end{array}$} & 5.1 Personne & $\begin{array}{l}\text { 5.1.2 Intégration sociale } \\
\text { 5.1.3 Construction d'identité } \\
\text { 5.1.4 Réalisation personnelle }\end{array}$ & $\begin{array}{l}5.1 .1 \text { "Ceux qui demandent l'aide d'un médecin sont } \\
\text { toujours angoissés (...) j'aimerais bien voir ma fille } \\
\text { aider les autres..." } \\
5.1 .2 \text { "(...) j'aimerais qu'elle ait des rapports ami- } \\
\text { caux et responsables avec les professeurs et camara- } \\
\text { des" " } \\
5.1 .3 \text { "Jaimerais qu'il ait plus de confiance en soi" } \\
5.1 .4 \text { "Avant tout, je voudrais qu'il soit un adulte } \\
\text { heureux" }\end{array}$ \\
\hline & 5.2 Étudiant & $\begin{array}{l}\text { 5.2.1 Niveau d'études } \\
5.2 .2 \text { Avenir } \\
\text { 5.2.3 Réussite scolairc } \\
\text { 5.2.4 Attitude face à l'étude }\end{array}$ & $\begin{array}{l}\text { 5.2.1 "Je veux qu'elle obtienne un diplôme supé- } \\
\text { rieur" } \\
5.2 .2 \text { "Ça dépend de ses propres capacités. parce } \\
\text { qu'on ne peut pas être tous docteurs ou ingenieurs" } \\
5.2 .3 \text { "Jc ne la pousse pas à être la meilleurc. Je ne } \\
\text { veux pas stimuler la compétition parce que, très sou- } \\
\text { vent, cela donne de mauvais résultats pour les jeunes" } \\
5.2 .4 \text { "Il faut absolument être un bon étudiant pour } \\
\text { devenir un bon professionnel, quel que soit le métier } \\
\text { qu on choisit" }\end{array}$ \\
\hline & $\begin{array}{l}5.3 \\
\text { Professionncl }\end{array}$ & $\begin{array}{l}\text { 5.3.1 Emploi satisfaisant } \\
\text { 5.3.2 Réussitc prolessionnele } \\
\text { 5.3.3 Groupe professionnel }\end{array}$ & $\begin{array}{l}5.3 .1 \text { "J'espère qu'il aura un métier et un style de vie } \\
\text { lui permettant un train de vie aisé... pas nécessairement } \\
\text { riche" } \\
5.3 .2 \text { "Je voudrais surtout qu'elle soit heureuse dans } \\
\text { sa vie professionnelle, qu'elle puisse réussir..." } \\
5.3 .3 \text { "J'aimerais qu'il suive un cours d'ingénieric } \\
\text { informatique ou èlectronique" }\end{array}$ \\
\hline \multirow{3}{*}{$\begin{array}{l}\text { 6. Perspectives } \\
\text { des enfants }\end{array}$} & $\begin{array}{l}6.1 \text { Axc } \\
\text { personnel }\end{array}$ & $\begin{array}{l}\text { 6.1.1 Spéciliécs } \\
\text { 6.1.2 Non spécifiées }\end{array}$ & $\begin{array}{l}\text { 6.1.1 "Je pense qu'il est assez déterminé par la } \\
\text { santé " } \\
\text { 6.1.2 "Tous les jours il arrive à la maison et parle } \\
\text { d'un projet différent, se posant des questions sur les } \\
\text { difficultés de réussir" }\end{array}$ \\
\hline & $\begin{array}{l}6.2 \text { Axe } \\
\text { parental }\end{array}$ & $\begin{array}{l}\text { 6.2.1 Spécifiées } \\
6.2 .2 \text { Non spécifiées }\end{array}$ & $\begin{array}{l}6.2 .1 \text { "J'aimerais le voir dans la recherche, dans le } \\
\text { domaine qu'il préfère" } \\
6.2 .2 \text { "J'ai horreur des hôpitaux, des malades et des } \\
\text { problèmes de santé... Serail-ce par hasard qu'il en ait } \\
\text { aussi ?" }\end{array}$ \\
\hline & $\begin{array}{l}6.3 \mathrm{Axe} \\
\text { social }\end{array}$ & $\begin{array}{l}\text { 6.3.1 Spéciliées } \\
\text { 6.3.2 Non spécifiées }\end{array}$ & $\begin{array}{l}\text { 6.3.1 "Il aime l'anglais, mais il croil que les Humani- } \\
\text { tés cela intéresse surtout les filles, done il n'a pas le } \\
\text { courage de suivre ce chemin " } \\
6.3 .2 \text { "Il demande quel salaire on peut obtenir dans } \\
\text { un certain métier... " }\end{array}$ \\
\hline
\end{tabular}

Grille de catégorisation des entretiens avec les parents (3/3)

Parent interview categorization

L'ensemble de ces résultats met en évidence l'importance des intentions parentales vis-àvis du développement vocationnel des enfants, aussi bien que l'importance qu'ils reconnaissent aux différents contextes, agents et activités. Les parents s'attribuent un rôle important, avouant toutefois des difficultés pour la mise en acte de pratiques éducatives spécifiques. En outre, bien que reconnaissant l'autonomie, les parents introduisent dans leurs discours des motifs et des objectifs personnels dans l'interprétation des projets de leurs enfants, sans toutefois s'en rendre compte.

En conclusion de l'analyse, on a essayé de trouver des supports théoriques et empiriques aux résultats qu'on avait obtenus, de façon à les intégrer dans un cadre conceptuel permettant de leur attribuer un sens psychologique. On a pris d'abord le modèle de Young et Friesen (1992) pour catégoriser les intentions parentales afin d'approfondir ce domaine qui se montrait assez central dans l'étude, mais qui ne semblait pas suffisamment clair. En fait, on a retrouvé chez ces parents portugais des catégories semblables aux catégories identifiées par ces auteurs, notamment: faire acquérir aux enfants compétences et valeurs, les protéger d'expériences désagréables, développer l'autonomie de pensée et d'action, faciliter les relations familiales et sociales, développer la responsabilité personnelle et le raisonnement moral, et aussi correspondre aux objectifs des parents.

Par ailleurs, les intentions parentales ne constituaient qu'une catégorie d'un ensemble beaucoup plus large d'éléments des discours des parents, concernant soit les agents du développement vocationnel des enfants, soit les objectifs, les moyens et les contextes de ce développement. Ces éléments semblent se rapprocher des composantes de la 
compétence de compréhension pratique identifiées par Ricoeur (cité par Polkinghorne, 1990) dans ses recherches phénoménologiques sur l'expérience de l'action, et utilisées par Young et collaborateurs dans leur approche de l'étude des carrières dans la perspective de la théorie de l'action (Young \& Friesen, 1990). À savoir :

- les actions sont accomplies dans le but d'obtenir des résultats, on peut donc leur attribuer des finalités, des objectifs ;

- les actions sont accomplies par des agents, c'est-à-dire par des personnes qui en prennent la responsabilité ;

- les actions sont associées à des raisons qui expliquent pourquoi elles sont accomplies, à des motifs plutôt reliés au sens qu'elles prennent pour les agents ;

- les actions ont lieu dans le cadre de systèmes physiques, les agents reconnaissant que les circonstances peuvent être, ou non, favorables aux résultats des actions ;

- les actions peuvent prendre différentes formes et expressions dans le cadre de l'interaction avec d'autres personnes, aussi bien que dans le recours à des moyens différents, qui peuvent rendre plus facile ou plus difficile leur accomplissement ;

- le résultat de l'action ouvre des voies de changement, en termes de connaissances, d'attitudes, de sentiments, d'opportunités.

Enfin, d'après les résultats de ces recherches, tout se passe comme si les parents portugais voulaient jouer un rôle important dans le développement vocationnel de leurs enfants, envisageant plusieurs expressions de communication et d'intervention familiale dans ce but, parlant facilement de leurs pensées et de leurs émotions concernant l'avenir qu'ils souhaitent pour leurs enfants et de ce qu'ils croient que leurs enfants souhaitent euxmêmes, attribuant au développement vocationnel un sens social important (Young, Valach \& Collin, 1996). Il parait donc légitime de prendre le cadre de la théorie de l'action pour soutenir, d'un point de vue conceptuel, les résultats des études portugaises, qu'il faut désormais poursuivre dans cette voie.

À ce moment de la recherche, on peut dire qu'on est arrivé à la mise au point d'une méthodologie qui semble pertinente pour l'intervention auprès des parents, concernant le développement vocationnel de leurs enfants. Le plan d'interview s'est montré facile à utiliser et capable de faire émerger un ensemble d'éléments importants repérés dans la théorie, les études empiriques et la pratique vocationnelle. De plus, l'analyse des données obtenues dans son application a permis de construire une grille cohérente pour guider, soit l'organisation et l'interprétation des discours des parents, soit une approche conceptuelle des psychologues conseillers dans leurs interventions de conseil ou de formation (Pinto \& Soares, 2002b). Notamment, la question « pourquoi » introduite dans le plan de l'interview semble créer des opportunités de confrontation qui aident les parents à clarifier idées, émotions et intentions, permettant aux psychologues de les aider à changer leurs comportements en fonction d'objectifs partagés avec leurs enfants (Young, Valach \& Collin 2002). 


\section{BIBLIOGRAPHIE}

Amatea, E. S., \& Cross, E. G. (1980). Going places : A career guidance program for high school students and their parents. Vocational Guidance Quarterly, 28, 274-282.

Astone, N. M., \& Mclanahan, S. S. (1991). Family structure, parental practices and high school completion. American Sociological Review, 56, 309-320.

Birk, J. M., \& Blimline, C. A. (1984). Parents as career development facilitators : an untapped resource for the counselor. School Counselor, 31, 310-317.

Bardin, L. (1977). Análise de Conteúdo. Lisboa : Edições Afrontamento.

Blake, J. (1985). Number of siblings and educational mobility. American Sociological Review, 50, 84-94.

Blustein, D. L., Devenis, L. E., \& Kidney, B. A. (1989). Relationship between the identity formation process and career development. Journal of Counseling Psychology, 36, 196-202.

Bordin, E. S. (1990). Psychodynamic model of career choice and satisfaction. In D. Brown \& L. Brooks (Eds.), Career choice and development (2 ${ }^{\text {nd }}$ ed.) (pp. 102-144). San Francisco : Jossey-Bass.

Bratcher, W. E. (1982). The influence of family on career selection: A family systems perspective. Psychological Association (30 ${ }^{\text {th }}$, New Orleans, L. A., April 19-21, 1984).

Cochran, L. (1985a). Career grid workbook. Richmond, British Columbia, Canada : Buchanan-Kells.

Cochran, L. (1985b). Parent career guidance manual. Richmond, British Columbia, Canada : Buchanan-Kells.

Cochran, L. (1985c). Planning workbook. Richmond, British Columbia, Canada : Buchanan-Kells.

Cochran, L., \& Amundson, N. (1985). Activity self-exploration workbook. Richmond, British Columbia, Canada : Buchanan-Kells.

Frost, F., \& Diamond, E. E. (1979). Ethnic and sex differences in occupational stereotyping by elementary school children. Journal of Vocational Behavior, 15, 43-54.

Gottfredson, L. S. (1981). Circumscription and compromise : A developmental theory of occupational aspirations. Journal of Counseling Psychology Monograph, 28, 545-579.

Gottfredson, L. S. (1996). Gottfredson's theory of circumscription and compromise. In D. Brown, L. Brooks \& Associates, Career choice and development ( $3^{\text {rd }}$ ed.) (pp. 179-232). San Francisco, California : Jossey-Bass.

Holland, J. L. (1959). A theory of vocational choice. Journal of Counseling Psychology, 6, 35-45.

Holland, J. L. (1973). Making vocational choices : A theory of careers. NJ : Prentice-Hall.

Hoyt, K. B. (1984). Helping parents understanding career education. Journal of Career Education, 10, 216-224.

Jacobsen, R. B. (1971). An exploration of parental encouragement as an intervening variable in occupational-educational learning of children. Journal of Marriage and Family, 33, 174-182. 
Krumboltz, J. D. (1996). A learning theory of career counseling. In M. L. Savickas and W. B. Walsh (Eds.), Handbook of career counseling theory and practice. Palo Alto, California : Davies-Black Publishing.

Lent, R. W., Brown, S. D., \& Hackett, G. (1996). Career Development from a Social Cognitive Perspective. In D. Brown, L. Brooks \& Associates (Eds.), Career choice and development ( $3^{\text {rd }}$ ed.) (pp. 373-421). San Francisco, California : Jossey-Bass.

Lofquist, L. H., \& Dawis, R. V. (1969). Adjustment to work, a psychological view of man's problems in a work-oriented society. New York : Appleton-Century-Crofts.

Lopez, F. G., \& Andrews, S. (1987). Career indecision : A family systems perspective. Journal of Counseling and Development, 65, 304-307.

McDaniels, C., \& Hummel, D. (1984). Parents and career education. Journal of Career Education, 10, 225-233.

Osipow, S. H. (1983). Theories of career development ( $3^{\text {rd }}$ ed.). Englewood Cliffs, NJ : Prentice Hall, Inc. Osipow, S. H. (1994). Moving career theory into twenty-first century. In M. L. Savickas \& R. W. Lent (Eds.), Convergence in career development theories. Implications for science and practice. Palo Alto, California : Consulting Psychologists Press.

Palmer, S., \& Cochran, L. (1988). Parents as agents of career development. Journal of Counseling Psychology, 35, 71-76.

Penick, N. I., \& Jepsen, D. A. (1992). Family functioning and adolescent career development. The Career Development Quarterly, 40, 208-222.

Pinto, H. R., \& Soares, M. C. (2000). Guião de entrevista a pais (versão para investigação). Lisboa : Instituto de Orientação Profissional.

Pinto, H. R., \& Soares, M. C. (2001). Influência parental na carreira : evolução de perspectivas na teoria, na investigação e na prática. Psychologica, 26, 135-149.

Pinto, H. R., \& Soares, M. C. (2002a). Influência parental e desenvolvimento vocacional. Relatório de investigação. Lisboa : Instituto de Orientação Profissional.

Pinto, H. R., \& Soares, M. C. (2002b). Influência parental no desenvolvimento vocacional dos adolescentes. Revista Portuguesa de Psicologia, 36, 111-137.

Poirier, J., Clapier-Valladon, S., \& Raybaut, P. (1993). Les récits de vie. Théorie et pratique. Paris : P.U.F. (1 ${ }^{\text {re }}$ éd. 1983).

Polkinghorne, D. (1990). Action theory approaches to career research. In R. Young \& W. Borgen (Eds.), Methodological approaches to the study of career (pp. 87-105). New York : Praeger.

Roe, A. (1957). Early determinants of vocational choice. Journal of Counseling Psychology, 4, 212-217.

Roe, A., \& Lunneborg, P. W. (1990). Personality development and career choice. In D. Brown \& L. Brooks (Eds.), Career choice and development ( $2^{\text {nd }}$ ed.) (pp. 68-101). San Francisco : Jossey-Bass.

Schulenberg, J. E., Vondracek, F. W., \& Crouter, A. C. (1984). The influence of the family on vocational development. Journal of Marriage and Family, 46, 129-143.

Soares, M. C. (1998). Influência parental no desenvolvimento da carreira. Estudo piloto sobre necessidades de formação de pais. Dissertação de Mestrado. Lisboa : Faculdade de Psicologia e de Ciências da Educação (mimeo).

Soares, M. C., \& Pinto, H. R. (1994). Questionário de avaliação de necessidades de formação de pais (versão para investigação). Lisboa : Faculdade de Psicologia e de Ciências da Educação. 
Soares, M. C., \& Pinto, H. R. (1997). Envolvimento dos pais no desenvolvimento da carreira. In H. Marchand \& H. R. Pinto (Eds.). Colóquio Familia : Contributos da Psicologia e das Ciências da Educação. Actas. Lisboa : Educa.

Stevens, P. (1989). Helping your child choose a career (1 ${ }^{\text {st }}$ ed.). Sydney : Centre for Worklife Counseling.

Super, D. E. (1957). Psychology of careers. New York : Harper.

Super, D. E. (1980). A life-span, life-space approach to career development. Journal of Vocational Behavior, 16, 282-298.

Super, D. E. (1990). A life-span, life-space approach to career development. In D. Brown \& L. Brooks (Eds.), Career choice and development ( $2^{\text {nd }}$ ed.) (pp. 197-261). San Francisco : Jossey-Bass.

Super, D. E., \& Thompson, A. S. (1979). A six-scale, two-factor measure of adolescent career of vocational maturity. Vocational Guidance Quarterly, 28, 6-15.

Thompson, A. S., \& Lindeman, R. H. (1984). Career Development Inventory. Volume 2 : Technical manual. Palo Alto : Consulting Psychologist Press, Inc.

Vodanovich, S. J., \& Kramer, T. J. (1989). An examination of the work values of parents and their children. The Career Development Quarterly, 37, 365-374.

Vondracek, F. W. (1990). A developmental-contextual approach to career development research. In R. A. Young \& W. A. Borgen (Eds.), Methodological approaches to the study of career (pp. 37-56). New York : Praeger.

Vondracek, F. W., \& Fouad, N. A. (1994). Developmental contextualism : An integrative framework for theory and practice. In M. L. Savickas \& R. W. Lent (Eds.), Convergence in career development theories. Implications for science and practice. Palo Alto, California : Counsulting Psychologists Press.

Young, R. A. (1993). Toward an understanding of joint action in career development. Discussion paper presented to the Third International Symposium on Career Development, Toronto. ON, August 19, University of British Columbia.

Young, R. A. (1994). Helping adolescents with career development : The active role of parents. The Career Development Quarterly, 42, 195-203.

Young, R. A., \& Borgen, W. A. (Eds.) (1990). Methodological approaches to the study of career. New York : Praeger.

Young, R. A., \& Friesen, J. D. (1990). Parental influences on career development : A research perspective. In R. A. Young \& W. A. Borgen (Eds.), Methodological Approaches to the Study of Career (pp. 147-162). New York : Praeger.

Young, R. A., \& Friesen, J. D. (1992). The intentions of parents in influencing the career development of their children. The Career Development Quarterly, 40, 198-207.

Young, R. A., Friesen, J. D., \& Borycki, B. (1994). Narrative structure and parental influence in career development. Journal of Adolescence, 17, 173-191.

Young, R. A., Friesen, J. D., \& Dillabough, J. M. (1991). Personal constructions of parental influence related to career development. Canadian Journal of Counseling, 25, 183-190.

Young, R. A., Friesen, J. D., \& Pearson, H. M. (1988). Activities and interpersonal relations as dimensions of parental behavior in the career development of adolescents. Youth and Society, 20, 29-45. 
Young, R. A., Valach, L., Ball, J., Paseluikho, M. A., Wong, Y. S., DeVries, R. J., McLean, H., \& Turkel, H. (2001). Career development in adolescence as a family project. Journal of Counseling Psychology, $48,190-202$.

Young, R. A., Valach, L., Ball, J., Turkel, H., \& Wong, Y. S. (2002). The family career development project in Chinese Canadian families. Journal of Vocational Behavior. In press.

Young, R. A., Valach, L., \& Collin, A. (1996). A Contextual Explanation of Career. In D. Brown, L. Brooks, \& Associates (Eds.), Career choice and development ( $3^{\text {rd }}$ ed.) (pp. 477-512). San Francisco, California : Jossey-Bass.

Young, R. A., Valach, L., \& Collin, A. (2002). A Contextual Explanation of Career. In D. Brown \& Associates, Career choice and development ( $4^{\text {th }}$ ed.) (pp. 206-252). San Francisco, California : JosseyBass.

\section{NOTES}

1. Les enfants avaient préalablement rempli la version portugaise du C.D.I.

\section{RÉSUMÉS}

On présente une synthèse des études portugaises concernant l'influence des parents sur le développement vocationnel des adolescents : la première étude, dans le cadre théorique de la maturité vocationnelle de Super, aboutit à un questionnaire utilisé avec 90 parents d'élèves de la $9^{\text {e }}$ année de scolarité; la deuxième étude propose une méthode d'analyse de contenu sur 8 entretiens de recherche. La troisième étude, suivant aussi une approche qualitative, présente les résultats de l'analyse de 64 entretiens avec des parents d'élèves de $6^{\mathrm{e}}$ et de $9^{\mathrm{e}}$ année de scolarité, interprétés dans le cadre de la théorie de l'action. En conclusion, on propose une méthodologie d'intervention avec desparents envisageant leur engagement dans le développement vocationnel de leurs enfants.

This article presents Portuguese research regarding parental influence on career development in adolescents. The first study used a questionnaire based on career maturity dimensions by Super taking a sample of 90 parents of $9^{\text {th }}$ grade students. The second study was a qualitative analysis of research interviews of a group of 8 parents. The third study, also qualitative, presented a description of results from 64 interviews with parents of $6^{\text {th }}$ and $9^{\text {th }}$ grade students and used action theory in their interpretation. To conclude, the author puts forward a method of working with the parents involved in the career development of their children, as a contribution to Portuguese studies in this field.

\section{INDEX}

Keywords : Action theory, Career development, Parental influence

Mots-clés : Développement vocationnel, Influence parentale, Théorie de l'action 


\section{AUTEURS}

\section{HELENA REBELO PINTO}

Helena Rebelo Pinto, docteur en psychologie, est professeur de psychologie de l'orientation à la Faculté de psychologie et d'éducation de l'Université de Lisbonne et directeur de l'Institut d'orientation professionnelle. Ses principaux thèmes de recherche, sur lesquelles elle a publié plusieurs travaux, sont le développement vocationnel des adolescents, notamment concernant l'influence parentale et la construction de programmes d'Éducation à la Carrière dans les écoles. Contact: Institut d'Orientation Professionnelle, Largo Trindade Coelho, 21, $1^{\circ}$ 1200-470 Lisboa Portugal. Courriel : Rebelo.Pinto@iop.ul.pt

\section{MARIA DA CONCEIÇÃO SOARES}

Maria da Conceição Soares, maître en psychologie, est conseillère d'orientation et chargée de recherche à l'Institut d'orientation professionnel de l'Université de Lisbonne. Ses principaux thèmes de recherche sont l'influence de la famille dans la construction de l'identité vocationnelle. 